\title{
The impact of COVID-19 lockdown on disordered eating behaviors: the mediation role of psychological distress
}

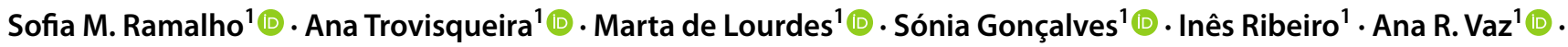 \\ Paulo P. P. Machado ${ }^{1}$ (D) Eva Conceição ${ }^{1}$ (i)
}

Received: 24 July 2020 / Accepted: 22 January 2021 / Published online: 13 March 2021

(c) The Author(s), under exclusive licence to Springer Nature Switzerland AG part of Springer Nature 2021

\begin{abstract}
Purpose This study aimed to explore the early associations between the experienced psychosocial impact of the COVID19 pandemic crisis during lockdown, depressive symptomatology, anxiety/stress levels, and disordered eating behaviors in adults during a first COVID-19 lockdown period.

Methods This was a community-based cross-sectional study assessing 254 Portuguese adults (82.7\% women; $35.82 \pm 11.82$ years) 1 week after the end of the first mandatory COVID-19 lockdown in Portugal. An online survey was conducted to evaluate psychological distress, disordered eating, and psychosocial impact of the COVID-19 pandemic. Pearson correlations and Structural Equation Modeling were performed.

Results Participants reported the presence of meal skipping (52.8\%), grazing eating behavior (80.9\%), overeating (81.0\%), loss of control over eating (47.2\%), and binge eating episodes (39.2\%) during lockdown. Structural equation modeling analyses, controlling for age and sex, indicated that there was a significant indirect effect of the experienced psychosocial impact of COVID-19 pandemic on disordered eating behaviors mediated through psychological distress.

Conclusion The psychosocial impact of the COVID-19 pandemic crisis may lead to disordered eating, and this relation may occur through the elevation of psychological distress. These findings can be used to inform interventions, to enhance mental health and manage disordered eating during similar future situations.

Level of evidence

V: cross-sectional descriptive study
\end{abstract}

Keywords COVID-19 lockdown $\cdot$ Eating behaviors $\cdot$ Psychological distress $\cdot$ Mediation $\cdot$ SEM

\section{Introduction}

The World Health Organization (WHO) declared the COVID-19 outbreak as a public health emergency of international concern in 2020 [1]. In this pandemic context, governments worldwide followed public health recommendations imposing lockdown and restrictions to curb the spread of this disease. These restrictive measures resulted in an unprecedented impact on live core domains, such as social and family interaction, employment, health care access, and finances.
Sofia M. Ramalho

sofia.mm.ramalho@gmail.com

Ana Trovisqueira

trovisqueiraana@gmail.com

Marta de Lourdes

martamagalhaeslourdes@outlook.com

Sónia Gonçalves

sgoncalves@psi.uminho.pt

Inês Ribeiro

nesnribeiro@gmail.com

\author{
Ana R. Vaz \\ anavaz@psi.uminho.pt \\ Paulo P. P. Machado \\ pmachado@psi.uminho.pt \\ Eva Conceição \\ econceicao@psi.uminho.pt \\ 1 Psychotherapy and Psychopathology Research Unit, \\ Psychology Research Centre, School of Psychology, \\ University of Minho, Campus Gualtar, 4710-057 Braga, \\ Portugal
}


In Portugal, the first COVID-19 case was confirmed on the 2nd of March of 2020. On the 18th of March, 16 days later, the state of emergency was declared for the first time imposing home confinement, prohibiting public gatherings, and closing public services/commercial establishments. At the end of the first COVID-19 emergency state (3rd May 2020), Portugal had 25,282 confirmed cases and 1043 deaths due to COVID-19 [2]. In spite of the reduction on the active cases during the summer season (June-September 2020), with the worsening of the global situation and to deal with the second wave of the COVID-19 pandemic in Portugal, the state of emergency was again declared (15 January 2021) imposing partial weekend lockdowns during November/ December 2020 and a second total lockdown with similar restrictions to the first one.

While these restrictive measures aimed to ensure social distancing and help to decrease the rate of COVID-19 infection, such limitations imposed a burden on the mental health of the general population that is now emerging, with reports of increased levels of stress, depression, anxiety, insomnia, feelings of loneliness, cases of post-traumatic stress and suicide [3-7]. In a study with the Portuguese population $25 \%$ of the adults reported moderate to severe symptoms of anxiety, depression and post-traumatic stress due to lockdown [8] and a recent study showed that higher levels of intolerance for uncertainty seemed to have a direct impact on mental wellbeing during the COVID-19 pandemic, with rumination and fear of COVID-19 mediating this association [9].

Moreover, results from an international online survey confirmed the negative effect of COVID-19 in dietary habits, specifically on unhealthy food consumption, loss of control over eating, meal skipping and snacking [10]. Nevertheless, up to date, the influence of the COVID-19 pandemic crisis on eating behaviors during lockdown is largely understudied. This is critical since the adverse mental health consequences related to COVID-19 pandemic can result in chronic psychopathology stimulating the upsurge of eating disorder risk and the exacerbation of symptoms in individuals previously diagnosed with an eating disorder [11-14]. Indeed, disordered eating behaviors, such as cognitive restraint, uncontrolled and emotional eating, have a link with eating disorders [11-14], but also with obesity [15-17].

Home confinement, imposed by mandatory lockdown, might have prompted the increase of food availability and generalized disorganized eating patterns [11, 14]. Rodgers et al. [13] proposed three pathways through which the COVID-19 pandemic, together with its associated high rates of psychological distress, may increase the risk of suffering from an eating disorder. The first path postulates that weight and shape concerns can increase due to the interferences in daily routines, outdoor activity restriction, and social distance restrictions, undermining eating disorder protective factors, such as social support and adaptive coping strategies. The second and third pathways are associated with the amplified exposure to anxiety-provoking media and with the hypothetical effects of health-related concerns on the adoption of restrictive dietary patterns, characterized by the exclusion of certain foods groups or/and reduced household food availability due to fear of leaving the house and contamination, respectively.

Rodgers et al. [13] proposal is supported by the literature linking stress, depression, anxiety, and other adverse mood states to a negative impact on eating behaviors [18-21]. Hence, in the scope of exploring the impact of COVID-19 pandemic on eating behaviors, psychological distress seems to assume particular relevance due to its shared risk factors and associations with disordered eating behaviors, such binge eating [18-21].

Lastly, the current uptrend in COVID-19 cases and the management difficulties in reducing the spread of the virus worldwide, highlight the possibility of multiple future lockdown periods and the importance of investigating how eating behaviors from the general population can be affected by public health restriction measures. In this context, it is mandatory to develop suitable lifestyle/dietary guidelines and identify high-risk groups to tailor effective interventions to minimize the impact of pandemic situations on eating behaviors [22].

Therefore, this study sought to explore the early associations between the experienced psychosocial impact of COVID-19 pandemic on the individuals' lives, depressive symptomatology, anxiety/stress levels, and disordered eating behaviors/attitudes in adults without a COVID-19 diagnosis during the first COVID-19 lockdown period in Portugal. Based on previous research showing associations between psychological distress and disordered eating behaviors [18-21], we aimed to test an atemporal mediation model [23] based on the hypothesis that the experienced psychosocial impact of COVID-19 pandemic is related to disordered eating behaviors through the mediation of experienced psychological distress during a first COVID-19 lockdown period.

\section{Methods}

\section{Participants}

This is a community-based cross-sectional study assessing adults from the general population without a diagnosis of COVID-19 1 week after the 46 days mandatory COVID-19 lockdown in Portugal (18th March to 3rd May 2020).

Participants' inclusion criteria: (1) aged between 18 and 68 years old; (2) living in Portugal during the COVID-19 lockdown. A total of 364 adults responded to our survey. Participants missing more than $20 \%$ of the items [24] on 
at least one of the variables under study were excluded $(n=95)$. Participants that have been diagnosed with COVID$19(n=15)$ were also excluded resulting in a final sample size of 254 participants.

\section{Procedure}

The data collection was exclusively online using Qualtrics ${ }^{\mathrm{XM}}$ : Online Survey Software. A non-probability consecutive sampling method was used. The study aims, participants' inclusion criteria and the link for accessing the anonymous online survey were publicized in social networks and academic/personal mailing lists. The survey took approximately $20 \mathrm{~min}$ to complete. Randomization of self-report measures presentation was used to reduce response bias. Participants provided their online consent, were informed about the risks/benefits of participation, and about the right to quit the survey at any time. The research team contact details were provided for participants' support regarding any concern/distress resulting from taking part in the survey.

Data collection started at May 11, 2020, 1 week after the end of the first mandatory COVID-19 lockdown in Portugal, and took place for 2 weeks under a state of calamity where the Portuguese population had the civic duty of home confinement, in spite of the reopening of some commercial establishments and public services. During this time, official data points to a national percentage of individuals in home confinement ranging from 47.4 to $48.1 \%$ (https://www.pse. pt/evolucao-confinamento-mobilidade/). This study was implemented in accordance with the Declaration of Helsinki and approved by the University ethics review board.

\section{Measures}

Sociodemographic and lifestyle questionnaire Participants answered about sociodemographic/lifestyle information's and social isolation conditions in the context of COVID19 pandemic, namely age, sex, education level, marital status, working status, household income, number of hours of sleep (weekdays/weekend) and physical activity ("In the past week, on how many days have you done a total of $30 \mathrm{~min}$ or more of physical activity, which was enough to raise your breathing rate." [25]).

Anthropometric data Weight (in kilogram) and height with no shoes (in centimeter) were self-reported. Body Mass Index (BMI) was computed for each participant and classified according to WHO BMI cut-offs [26]. Perception of weight change (increase, decrease or maintenance) during the COVID- 19 lockdown period was also assessed.

Disordered Eating Behaviors Screening Questionnaire This questionnaire was designed in the scope of this study and includes five questions (one per eating behavior) concerning the frequency of the following disordered eating behaviors in the last month (from 0 to 31 days): meal skipping, grazing eating behavior, loss of control over eating, objective overeating and binge eating episodes. Explicitly "Thinking about the last month (31 days), how many days did you (...)":

1. “(...) eat repetitively small or modest amounts of food between meals without planning it throughout the day?";

2. “(...) skip meals (e.g., breakfast, morning snack, lunch, etc.)?";

3. “(...) feel you ate too much or more than you should (for example, repeating the plate, continuing to eat after you were already feeling full, or overeating without being hungry)?";

4. "(...) feel that you ate compulsively or that you had lost control over what you were eating (e.g., not being able to resist eating, or not being able to stop after starting to eat)?";

5. “(...) feel that you ate compulsively, uncontrollably eating excessively large amounts of food for the circumstances (e.g., eating twice or more than someone else would eat in the same situation)?".

Coronavirus Impact Scale (CIS) [27] It comprises 10 questions rated on a 4 -point Likert scale $(0=$ none/no change to $3=$ severe) that follow the probe statement: "Please rate to what extent the COVID-19 pandemic changed your life in each of the following areas": routines, family income/ employment, food and mental health care access, access to social support, experience of stress related to COVID19 pandemic, stress/family discord, personal diagnosis of coronavirus, immediate/extended family members and close friends diagnosed with COVID-19. The final item is an open question about other ways the pandemic might have affected the respondent daily life. In this study we used the overall COVID-19 psychosocial impact score that was computed as the direct sum of participants answers to all 10 closedended questions, as suggested by the original authors. This scale was adapted from English to Portuguese following a translation-back-translation procedure [28]. Preliminary analyses indicate good reliability (McDonald's $\omega$ for this sample: total score $=0.77$ ).

Depression Anxiety Stress Scales (DASS-21) [29] This instrument has 21 items and generates three subscales: depression, anxiety and stress. The score of each subscale ranges between 0 and 21 points. Higher scores correspond to more negative affective states in adults (McDonald's $\omega$ for this sample: Depression Scale $=0.91$; Anxiety Scale $=0.88$; Stress Scale $=0.89$ ). Participants in this study completed the Portuguese version of the DASS-21 [30].

Three-Factor Eating Questionnaire Revised 21-item version (TFEQ-R21) [31] It consists of 21 items divided 
into 3 scales, corresponding to restrained eating, emotional eating, and uncontrolled eating. Scores for each scale vary between 0 and 100 . Higher scores are indicative of greater cognitive restraint, uncontrolled, emotional eating (McDonald's $\omega$ for this sample: uncontrolled eating $=0.89$; emotional eating $=0.93$; cognitive restraint $=0.85$ ). Participants in this study completed the Portuguese version of the TFEQ-R21 [32].

\section{Statistical analyses}

Descriptive statistics were conducted to describe participants' sociodemographic, anthropometric, lifestyle and disordered eating behavior characteristics. Missing value analysis indicated that DASS-21 and TFEQ-R21 missing value percentages varied between 2.6 and $4.4 \%$. Little's MCAR test showed that data were missing completely at random (DASS-21: $\chi^{2}(20)=12.19, p=0.909$; TFEQ$\left.\mathrm{R} 21: \chi^{2}(1)=1.46, p=0.228\right)$ and item-level missing data were handled by mean imputation. Kurtosis and skewness values for main variables were below $|1.0|$ denoting a normal distribution. The IBM ${ }^{\circledR}$ SPSS $^{\circledR}$ Statistics 25.0 (SPSS Inc., Chicago, IL) was used. Additionally, McDonald's $\omega$ was calculated as an estimate of scale reliability for all the self-report measures using JASP version 0.12.2 (JASP Team University of Amsterdam, Amsterdam, The Netherlands).

First, Pearson's correlation coefficients were used to investigate associations between variables under study and to define the final mediation model to test. Subsequently, to explore the relationships among psychosocial COVID-19 pandemic impact, psychological distress variables (anxiety, depression and stress) and disordered eating behaviors (uncontrolled eating and emotional eating) mediation effects were tested with a Structural Equation Model (SEM) using Maximum Likelihood Estimation in IBM $^{\circledR}$ SPSS $^{\circledR}$ Amos $^{\text {TM }} 22.0$ after the measurement model validation [33]. The bias-corrected bootstrapping procedure (95\% confidence) was applied to compute the standard errors of the indirect effects. Model fit was examined using the following fit indexes: normed chi-square (CMIN/DF) with values $<5$ representing acceptable fit; Tucker-Lewis Index (TLI), Incremental Fit Index (IFI) > 0.90; Comparative Fit Index (CFI) > 0.95; Root Mean Square Error of Approximation index $($ RMSEA) $\leq 0.08$ [34, 35]. Age and sex were included as covariables. $p$ values $<0.05$ were considered significant. As recommended by Winer et al. [23], the term "atemporal" was used through the paper to emphasis that the mediational effects derived from a cross-sectional design, and therefore do not imply causation.

\section{Results}

\section{Participants}

A total of 254 adults participated in this study. Age ranged from 18 to 68 years $(M=35.82, \mathrm{SD}=11.82), 210$ were women $(82.7 \%)$. Table 1 shows the detailed participants' characterization. The majority of participants, 152 have completed a master's degree/Ph.D. (59.8\%). Out of the 254 respondents, 121 participants $(47.7 \%)$ were married or lived together with a romantic partner, $120(47.2 \%)$ were single, and 13 divorced/separated (5.1\%). Regarding the employment status, 139 participants reported to be employed in teleworking $(54.7 \%)$, with $33.5 \%(n=85)$ reporting a decrease in total family income due to COVID-19 pandemic. During this period, $16.5 \%(n=42)$ of participants stated a total family income inferior to $1000 €$ in the previous month, $41.4 \%$

Table 1 Participants' sociodemographic, anthropometric and lifestyle characterization

\begin{tabular}{|c|c|c|c|}
\hline & $n$ & $\%$ & Mean (SD) \\
\hline Age, years & & & $35.82(11.82)$ \\
\hline \multicolumn{4}{|l|}{ Sex } \\
\hline Female & 210 & 82.7 & \\
\hline Male & 44 & 17.3 & \\
\hline \multicolumn{4}{|l|}{ Educational attainment } \\
\hline$\leq$ High school degree & 33 & 13.0 & \\
\hline$\leq$ Bachelor's degree & 69 & 27.2 & \\
\hline$\geq$ Master's degree/Ph.D & 152 & 59.8 & \\
\hline \multicolumn{4}{|l|}{ Marital status } \\
\hline Single & 120 & 47.2 & \\
\hline Married/living together & 121 & 47.7 & \\
\hline Divorced/separated & 13 & 5.1 & \\
\hline BMI kg/m² (self-reported) & & & $4.08(4.57)$ \\
\hline \multicolumn{4}{|l|}{ BMI status-WHO } \\
\hline Underweight & 16 & 6.3 & \\
\hline Normal weight & 154 & 60.6 & \\
\hline Overweight & 56 & 22.0 & \\
\hline Obesity & 28 & 11.0 & \\
\hline \multicolumn{4}{|c|}{ Perception of weight change during COVID-19 lockdown } \\
\hline Weight increase & 97 & 38.2 & \\
\hline Weight decrease & 40 & 15.7 & \\
\hline Weight maintenance & 117 & 46.1 & \\
\hline Number of hours of sleep (weekday) & & & $7.24(1.12)$ \\
\hline Number of hours of sleep (weekend day) & & & $7.89(1.38)$ \\
\hline $\begin{array}{l}\text { Physical activity (number of } \\
\text { days } \geq 30 \mathrm{~min} \text { ) }\end{array}$ & & & $2.24(2.26)$ \\
\hline
\end{tabular}

$N=254$

$B M I$ body mass index, $S D$ standard deviation

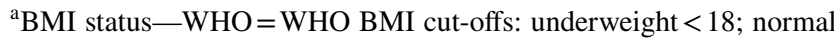
weight $\geq 18<25$; overweight $=\geq 25$; obesity $=\geq 30$ 
$(n=105)$ between $1000 €$ and $2000 €$, and $42.1 \%(n=107)$

reported a total family income superior to $2000 €$.

Participants' mean self-reported BMI was $24.08 \mathrm{~kg} /$ $\mathrm{m}^{2}(\mathrm{SD}=4.57), 22.0 \%(n=56)$ had overweight and $11.0 \%$ $(n=28)$ obesity (according to WHO cutoffs [26]). Considering the perception of the respondents regarding weight variation during the lockdown period, 97 individuals (38.2\%) reported an increase in weight, and $40(15.7 \%)$ participants reported a decrease in weight in the same period. Regarding lifestyle habits during the first COVID-19 lockdown, participants stated to sleep, on average, $7.24(\mathrm{SD}=1.12)$ hours per night on weekdays and $7.89(\mathrm{SD}=1.38)$ hours on weekends. Moreover, participants reported practicing, on average, a total of 30 min or more of physical activity (enough to raise breathing rate) in $2.24(\mathrm{SD}=2.26)$ days per week.

Table 2 illustrates the presence/absence and monthly frequency (number of days over the last month) of the following disordered eating behaviors during the first COVID-19 lockdown: meal skipping, grazing eating behavior, loss of control over eating, objective overeating and binge eating episodes.

\section{Psychosocial impact of COVID-19 pandemic}

Detailing the COVID-19 pandemic psychosocial impact, $59.1 \%$ individuals $(n=151)$ described a serious change on

Table 2 Presence and monthly frequency (number of days in the previous month) of disordered eating behaviors during COVID-19 lockdown

\begin{tabular}{|c|c|c|c|}
\hline & $n$ & $\%$ & Mean (SD) \\
\hline Skipping meals (no. of days) & & & $5.08(7.96)$ \\
\hline Absence & 120 & $47.2 \%$ & \\
\hline Presence (at least 1 day) & 134 & $52.8 \%$ & \\
\hline Grazing eating behavior $^{\mathrm{a}}$ (no. of days) & & & $10.16(9.72)$ \\
\hline Absence & 48 & $19.1 \%$ & \\
\hline Presence (at least 1 day) & 203 & $80.9 \%$ & \\
\hline $\begin{array}{l}\text { Objective overeating episode }{ }^{\mathrm{a}} \text { (no. of } \\
\text { days) }\end{array}$ & & & $7.85(8.38)$ \\
\hline Absence & 48 & $19.0 \%$ & \\
\hline Presence (at least 1 day) & 205 & $81.0 \%$ & \\
\hline $\begin{array}{l}\text { Loss of control over eating episode }{ }^{\mathrm{a}} \text { (no. } \\
\text { of days) (regardless the amount of food } \\
\text { ingested) }\end{array}$ & & & $3.88(6.69)$ \\
\hline Absence & 132 & $52.8 \%$ & \\
\hline Presence (at least 1 day) & 118 & $47.2 \%$ & \\
\hline Binge eating episode ${ }^{\mathrm{a}}$ (no. of days) & & & $3.00(5.89)$ \\
\hline Absence & 152 & $60.8 \%$ & \\
\hline Presence (at least 1 day) & 98 & $39.2 \%$ & \\
\hline
\end{tabular}

$N=254$

$S D$ standard deviation

${ }^{a}$ There are some missing values in these variables daily routines with changes on more than three life domains (e.g., work, social life, education, hobbies, etc.), $28.74 \%$ $(n=73)$ reported mild to serious difficulties in food access, and $21.3 \%(n=54)$ stated mild to moderate changes in medi$\mathrm{cal} /$ mental health care access with appointments moved to telehealth or delays/cancellations in appointments/getting prescriptions. Access to in-person extended family and nonfamily social support was also limited for $77.2 \%(n=196)$ of the sample during the lockdown. Most participants $(n=223$, $87 \%$ ) reported experiencing mild to severe stress related to COVID-19 pandemic (worries and/or stress-related symptoms $)$ and $52 \%(n=132)$ mild to severe family discord, with family members frequently engaged in arguments and/or physical fights. Just ten (3.9\%) individuals stated to have at least one immediate family member diagnosed with COVID-19, and 36 (14.17\%) reported to have at least one extended family member and/or close friend diagnosed with COVID-19.

\section{Associations between the experienced psychosocial impact of COVID-19 pandemic, psychological distress, and disordered eating behaviors during lockdown}

Table 3 presents the correlations between sociodemographic and anthropometric variables, disordered eating behaviors, psychological distress and psychosocial impact of COVID19. Results suggest that the experience of a higher psychosocial impact of COVID-19 pandemic during lockdown was significantly correlated with being women and young. Moreover, higher psychosocial impact of COVID-19 pandemic was positively associated with depressive symptomatology, anxiety, and stress levels as well with uncontrolled and emotional eating. There were no statistically significant associations between the psychosocial impact of COVID-19 pandemic with BMI or with cognitive restraint.

\section{The mediation role of psychological distress}

Existing research supports an association between psychological distress and disordered eating behaviors [18-21], and our results showed correlations between psychosocial impact of COVID-19 pandemic during lockdown, depressive symptomatology, anxiety/stress levels, uncontrolled and emotional eating (Table 3). Based on these findings, we hypothesized a mediation model in which the experienced psychosocial impact of COVID-19 pandemic is associated with disordered eating behaviors (uncontrolled and emotional eating) through (mediated by) the experienced psychological distress (depressive symptomatology, anxiety, stress levels). Structural equation modeling was used to test this atemporal association model [23]. 
Table 3 Correlations between the psychosocial impact of COVID-19 during lockdown and age, sex, BMI, psychological distress variables, and disordered eating behaviors

\begin{tabular}{|c|c|c|c|c|c|c|c|c|c|c|c|}
\hline & & 1 & 2 & 3 & 4 & 5 & 6 & 7 & 8 & 9 & 10 \\
\hline 1 & COVID-19 Psychosocial Impact (CIS) & - & & & & & & & & & \\
\hline 2 & Age & $-0.22 * * *$ & - & & & & & & & & \\
\hline 3 & Sex & $-0.15^{*}$ & $17 * *$ & - & & & & & & & \\
\hline 4 & BMI & -0.03 & $0.15^{*}$ & $0.13^{*}$ & - & & & & & & \\
\hline 5 & Depressive symptomatology (DASS-21) & $0.34 * * *$ & $-0.19 * *$ & -0.03 & 0.09 & - & & & & & \\
\hline 6 & Anxiety (DASS-21) & $0.34 * * *$ & $-0.19 * *$ & $-0.13^{*}$ & 0.02 & $0.66 * * *$ & - & & & & \\
\hline 7 & Stress (DASS-21) & $0.45^{* * *}$ & $-0.24 * * *$ & $-0.22 * * *$ & 0.05 & $0.63 * * *$ & $0.74 * * *$ & - & & & \\
\hline 8 & Uncontrolled eating (TFEQ-R21) & $0.18^{* *}$ & $-0.12^{*}$ & $-0.14^{*}$ & $0.23^{* * *}$ & $0.21 * *$ & $0.22 * * *$ & $0.26 * * *$ & - & & \\
\hline 9 & Emotional eating (TFEQ-R21) & $0.23 * * *$ & -0.12 & $-0.16^{*}$ & $0.22 * * *$ & $0.23 * * *$ & $0.21 * *$ & $0.30 * * *$ & $0.71 * * *$ & - & \\
\hline 10 & Cognitive restraint (TFEQ-R21) & 0.08 & 0.05 & -0.10 & $0.14 *$ & 0.00 & 0.02 & 0.03 & 0.04 & 0.10 & - \\
\hline
\end{tabular}

$N=254 ;$ Pearson's correlation coefficients

$B M I$ body mass index (calculated from self-reported weight/height), CIS Coronavirus Impact Scale, DASS-21 Depression Anxiety Stress Scales, $T F E Q-R 21$ Three-Factor Eating Questionnaire Revised 21-item version

${ }^{*} p<0.05 ; * * p<0.01 ; * * * p<0.001$

First, the hypothesized measurement model was tested using confirmatory factor analysis [33] resulting in a good fit to the data, CMIN/DF $=1.499, \mathrm{CFI}=0.99$, RMSEA $=0.045$ (90\% CI 0.00, 0.10). These values suggest that the loadings of the measured variables on the two latent variables were significant $(p<0.001)$ and that the latent variables (psychological distress and disordered eating behaviors) were adequately measured by their corresponding indicators [Psychological Distress: depressive symptomatology, anxiety/stress levels (DASS-21 scales); disordered eating behaviors: uncontrolled and emotional eating (TFEQ-R21 scales)].
The mediation model produced goodness-of-fit indexes that suggest a good fit to the data: CMIN/DF $=2.84$, $\mathrm{CFI}=0.95, \mathrm{TLI}=0.92, \mathrm{IFI}=0.95, \mathrm{RMSEA}=0.08(90 \% \mathrm{CI}$ $0.057,0.114)$. Sex and age were specified as covariates in the model based on the previous correlation analysis (Table 3), indicating its significant associations with the variables in the model. Mediation model parameters are presented in Fig. 1.

There was a full mediation between psychosocial impact of COVID-19 pandemic on disordered eating behaviors through psychological distress $[(b=0.97(\mathrm{CI}-0.71,2.60)$, $\beta=0.10, p=0.262$ )]. The indirect effect (psychosocial impact of COVID-19 pandemic psychological distress
Fig. 1 Graphic representation of the mediation model: the mediating role of psychological distress in the relation between the experienced psychosocial impact of COVID-19 pandemic and disordered eating behaviors during lockdown. Estimated standardized coefficients. All endogenous variables were associated with errors; $* * * p<0.001$

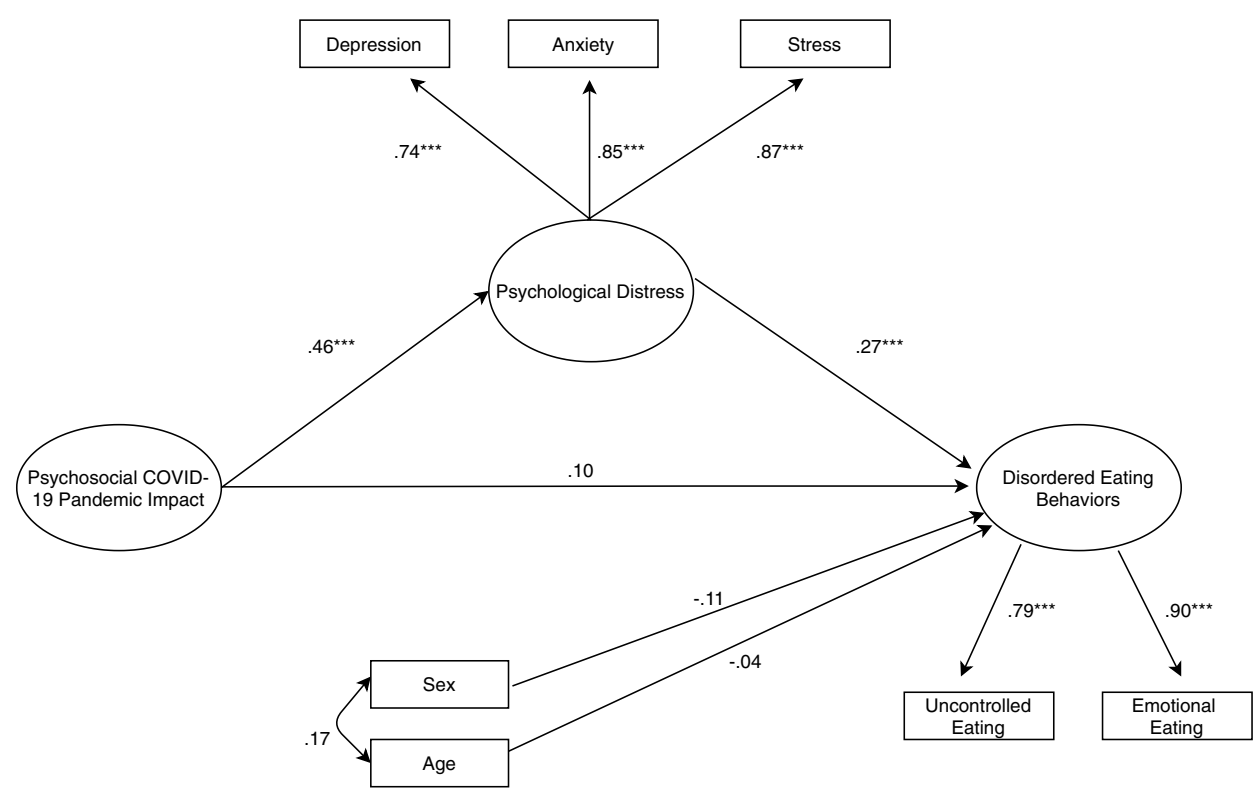


disordered eating) was significant $[(b=1.21$ (CI 0.44, 2.38), $\beta=0.12, p<0.003)]$. Finally, the total effect of psychosocial impact of COVID-19 pandemic during lockdown on disordered eating was also significant $[(b=2.17$ (CI $0.53,3.69$ ), $\beta=0.22, p<0.013)]$. These results suggest a significant and positive mediating effect of psychological distress.

\section{Discussion}

To our knowledge, this was the first study to examine the early relationships between psychosocial impact of COVID19 pandemic and disordered eating behaviors during the first lockdown period in a community sample in Portugal. We found that the change imposed by the psychosocial impact of COVID-19 lockdown on disordered eating behaviors was significantly associated with psychological distress. More importantly, the association between the psychosocial impact of COVID-19 and disorder eating (specifically, emotional and uncontrolled eating) seems to be better explained by the experienced psychological distress (depression, anxiety and stress) during this period. Our findings suggest that individuals who experience notable changes in a variety of domains in their daily life will experience increased psychological distress, which, in turn, results in more disordered eating. Despite being hypothesized by previous authors [13, 14], this is the first study to bring support for this association. In this context, eating may serve as a means to cope with the adverse psychological effects of COVID-19 pandemic during the lockdown [36, 37].

Results from the correlational analysis suggested that the experience of a higher psychosocial impact of COVID19 pandemic during lockdown was higher in women and in younger ages. However, these variables were not significantly linked with any variable in the mediation model suggesting that sex and age do not impact the psychological distress mediating effect. Some studies with the general population, including the Portuguese population [8], have shown that the mental health consequences of the COVID19 pandemic are more marked in women [38, 39] and at younger ages (18-35 years old) [40, 41]. Nevertheless, these associations are not consistently reported throughout the literature [41] highlighting the need for more studies about the effect of age and sex in the COVID-19 psychosocial impact and its associations with disordered eating in different cultures and across the lifespan.

In spite of the interventive efforts of the Portuguese authorities to mitigate the consequences of the COVID19 pandemic on dietary behavior/habits and to support an adequate nutritional status in higher-risk groups/COVID19 patients [42], about $38.2 \%$ of participants reported a perceived increase in weight due to their first COVID-19 lockdown period. This percentage is slightly superior to the $26.4 \%$ observed in the Portuguese population [43], but it is still a significant figure. Although this is a self-report of perceived weight variations about the general population, it calls for the attention to the impact of lockdown on overweight and obesity rates [44]. Moreover, the perceived weight gain on itself may indicate underlying disordered eating attitudes/behaviors making it a target for clinical attention.

For instance, participants in our study reported the presence of a variety of disordered eating behaviors, such as skipping meals (52.8\%), grazing eating behavior $(80,9 \%)$, overeating (81.0\%), loss of control over eating (47.2\%), and binge eating episodes (39.2\%). These figures point to a notable presence of unhealthy dietary patterns, during the first lockdown in the Portuguese community population. This is also supported by the national report on the changes in food consumption during the COVID-19 lockdown [43], changes prominently associated with obesity that seems to be characterized by the consumption of large portions, snacking, and loss of control eating. In spite of the lack of a pre-COVID-19 pandemic assessment, a study with the Australian population suggested an increase of $27.6 \%$ in food restriction and $34.6 \%$ in binge eating behaviors of the adult population, when compared with pre-pandemic period, illustrating the impact of lockdown periods on eating behaviors [14].

Individuals with obesity or problematic eating behaviors can show compromised social-emotional communication and poor insight regarding their illness which can delay help-seeking behaviors [11]. In this scope, the tested model can significantly inform intervention approaches designed to mitigate the short and long-term consequences of COVID-19 pandemic on obesity, creating a groundwork for pandemic situations involving lockdown and social isolation. It is of major importance to guarantee that COVID-19 pandemic does not inflate rates of obesity, which is not only associated with exacerbated manifestation of COVID-19 [45] but is also considered an epidemic [44]. Moreover, telehealth $/ m$ health approaches are promising strategies to address the modifiable domains that account for the psychosocial impact of COVID-19 pandemic (e.g. stress, family discordance, mental health care access, etc.), and decrease psychological distress and disordered eating during lockdown without a rise in the risk of infection [46].

This study brings attention for the fact that individuals in crisis situations might experience long-lasting psychological trauma and "emotional contagion" (the spread of affect and mood states through populations by exposure) [47]. Plus, depressive states seem to be linked to peripheral inflammation markers in COVID-19 quarantined patients, and consequently to a poorer prognosis [48].

To the best of our knowledge, this is the first study that focuses on the COVID-19 lockdown early impact on eating behaviors in Portugal. Additionally, data were collected in 
the early stage of calamity period in Portugal, just after a 3 consecutive periods of emergency state enabling the evaluation of the immediate impact of the first COVID-19 lockdown period on eating behaviors.

Yet, this study is limited due to the high proportion of highly educated women in the sample which prevents broader generalizations to more diverse populations, and also to its cross-sectional nature that inhibits causal hypotheses to be tested. Even though, we proposed to test an atemporal mediation model [23] that do not imply causation to avoid potential misinterpretation and literature supports the validation of the mathematical methods underlying mediation with cross-sectional data [49]. The limited scientific evidence about the effects of COVID-19 lockdown on eating behaviors reinforces the relevance and potential theoretical contribution of the tested model. Notwithstanding, future work should examine these variables longitudinally to confirm causality in a sample with a reduced overrepresentation of women with higher education. Also, exploring other assessment approaches, such as Ecological Momentary Assessment (EMA), to verify the temporal sequence of psychological distress and disordered eating behaviors [50] and its associations with emotional regulation strategies during lockdown periods. In addition, information about the frequency of help requests to private/public healthcare services related to disordered eating behaviors will be valuable to reinforce these study results.

On whole, the findings presented, contribute to understanding the impact of lockdowns on individuals' disordered eating behaviors and to inform the development of innovative interventions strategies aimed at preventing and reducing the early adverse effects of lockdowns. Since second lockdowns are a possibility and can be recurrent, such as in the Portuguese case.

\section{What is already known on this subject?}

To best of our knowledge, this is the first study to examine the relationship between psychosocial impact of COVID-19 pandemic and disordered eating behaviors during the first lockdown period in a Portuguese community sample.

\section{What this study adds?}

The results of this study showed that the change imposed by the psychosocial impact of a first COVID-19 lockdown on disordered eating behaviors was significantly associated with psychological distress. Findings highlight the importance of designing interventions to mitigate the short- and long-term consequences of COVID-19 pandemic on eating behaviors/ disorders, creating a groundwork for future situations involving lockdown and social isolation.

Funding This research was partially conducted at Psychology Research Centre (PSI/01662), University of Minho, through support from the Portuguese Foundation for Science and Technology and the Portuguese Ministry of Science, Technology and Higher Education (UID/ PSI/01662/2019), through the national funds (PIDDAC), by Grants to Paulo PP Machado (POCI-01-0145-FEDER-028145), Eva Conceição (IF/01219/ 2014 and POCI-01-0145-FEDER-028209), Ana Trovisqueira (SFRH/BD/136224/2018), and Marta de Lourdes (SFRH/ $\mathrm{BD} / 146470 / 2019)$. The funding body had no role in the design, collection, analysis, and interpretation of data; the writing of the manuscript; or the decision to submit the manuscript for publication.

Availability of data and materials The data that support the findings of this study are available from the corresponding author upon reasonable request.

\section{Compliance with ethical standards}

Conflict of interest The authors declare that they have no competing interest.

Ethics approval Ethical approval was obtained from the University of Minho Ethics Review Board-Subcommission of Social and Human Sciences (SECSH) (CEICSH 040/2020).

Informed consent Informed consent was obtained from all individual participants included in the study.

\section{References}

1. World Health Organization (2020) Coronavirus disease 2019 (COVID-19) situation report-51. World Health Organization. https://apps.who.int/iris/handle/10665/331475. Accessed 3 Feb 2021

2. Direção Geral da Saúde (2020) Relatório de Situação no 062 I 03/05/2020. Direção Geral da Saúde. https://covid19.min-saude. pt/wp-content/uploads/2020/05/62_DGS_boletim_20200503_V2. pdf. Accessed 3 Feb 2021

3. Bhuiyan AKMI, Sakib N, Pakpour AH et al (2020) COVID-19-related suicides in Bangladesh due to lockdown and economic factors: case study evidence from media reports. Int J Ment Health Addict. https://doi.org/10.1007/s11469-020-00307-y

4. de Carvalho PM, Moreira MM, de Oliveira MNA et al (2020) The psychiatric impact of the novel coronavirus outbreak. Psychiatry Res 286:1-2. https://doi.org/10.1016/j.psychres.2020.112902

5. Torales J, O'Higgins M, Castaldelli-Maia JM, Ventriglio A (2020) The outbreak of COVID-19 coronavirus and its impact on global mental health. Int J Soc Psychiatry 66:317-320. https://doi.org/ 10.1177/0020764020915212

6. Brooks SK, Webster RK, Smith LE et al (2020) The psychological impact of quarantine and how to reduce it: rapid review of the evidence. Lancet 395:912-920. https://doi.org/10.1016/S01406736(20)30460-8

7. Arslan G, Yıldırım M, Tanhan A et al (2020) Coronavirus stress, optimism-pessimism, psychological inflexibility, and psychological health: psychometric properties of the coronavirus stress 
measure. Int J Ment Health Addict. https://doi.org/10.1007/ s11469-020-00337-6

8. de Almeida TC, Heitor MJ, Santos O et al (2020) Saúde mental em tempos de pandemia - SM-COVID-19: relatório final. Instituto Nacional de Saúde Doutor Ricardo Jorge, Lisbon

9. Satici B, Saricali M, Satici SA, Griffiths MD (2020) Intolerance of uncertainty and mental wellbeing: serial mediation by rumination and fear of COVID-19. Int J Ment Health Addict. https://doi.org/10.1007/s11469-020-00305-0

10. Ammar A, Brach M, Trabelsi K et al (2020) Effects of COVID19 home confinement on eating behaviour and physical activity: results of the ECLB-COVID19 international online survey. Nutrients 12:1583. https://doi.org/10.3390/nu12061583

11. Fernández-Aranda F, Casas M, Claes L et al (2020) COVID19 and implications for eating disorders. Eur Eat Disord Rev 28:239-245. https://doi.org/10.1002/erv.2738

12. Shah M, Sachdeva M, Johnston H (2020) Eating disorders in the age of COVID-19. Psychiatry Res 290:113122. https://doi. org/10.1016/j.psychres.2020.113122

13. Rodgers RF, Lombardo C, Cerolini S et al (2020) The impact of the COVID-19 pandemic on eating disorder risk and symptoms. Int J Eat Disord 53:1166-1170. https://doi.org/10.1002/ eat. 23318

14. Phillipou A, Meyer D, Neill E et al (2020) Eating and exercise behaviors in eating disorders and the general population during the COVID -19 pandemic in Australia: initial results from the COLLATE project. Int J Eat Disord 53:1158-1165. https://doi. org/10.1002/eat.23317

15. Pinto-Bastos A, Ramalho S, Conceição E, Mitchell E (2015) Disordered eating in obesity. In: Ahmad SI, Imam SK (eds) Obesity: a practical guide. Springer International Publishing, Switzerland

16. McCuen-Wurst C, Ruggieri M, Allison KC (2018) Disordered eating and obesity: associations between binge-eating disorder, night-eating syndrome, and weight-related comorbidities. Ann NY Acad Sci 1411:96-105. https://doi.org/10.1111/nyas.13467

17. Nightingale BA, Cassin SE (2019) Disordered eating among individuals with excess weight: a review of recent research. Curr Obes Rep 8:112-127. https://doi.org/10.1007/s13679-019-00333-5

18. Fletcher B, Kupshik GA, Uprichard S et al (2008) Eating disorders and concurrent psychopathology: a reconceptualisation of clinical need through Rasch analysis. Eur Eat Disord Rev 16:191-198. https://doi.org/10.1002/erv.833

19. Rosenbaum DL, White KS (2015) The relation of anxiety, depression, and stress to binge eating behavior. J Health Psychol 20:887898. https://doi.org/10.1177/1359105315580212

20. Royal JD, Kurtz JL (2010) I ate what?! The effect of stress and dispositional eating style on food intake and behavioral awareness. Pers Individ Differ 49:565-569. https://doi.org/10.1016/j. paid.2010.04.022

21. Yannakoulia M, Panagiotakos DB, Pitsavos C et al (2008) Eating habits in relations to anxiety symptoms among apparently healthy adults. A pattern analysis from the ATTICA study. Appetite 51:519-525. https://doi.org/10.1016/j.appet.2008.04.002

22. Tanhan A, Yavuz KF, Young JS et al (2020) A proposed framework based on literature review of online contextual mental health services to enhance wellbeing and address psychopathology during COVID-19. Electron J Gen Med 17:2516-3507. https://doi. org/10.29333/ejgm/8316

23. Winer ES, Cervone D, Bryant J et al (2016) Distinguishing mediational models and analyses in clinical psychology: atemporal associations do not imply causation. J Clin Psychol 72:947-955. https://doi.org/10.1002/jclp.22298

24. Peng C, Harwell M, Liou S, Ehman L (2006) Advances in missing data methods and implications for educational research. In: Sawilowsky S (ed) Real data analysis (quantitative methods in education and the behavioral sciences: issues, research, and teaching). Information Age Pub, New York, pp 31-78

25. Milton K, Bull FC, Bauman A (2011) Reliability and validity testing of a single-item physical activity measure. Br J Sports Med 45:203-208. https://doi.org/10.1136/bjsm.2009.068395

26. World Health Organization (2020) Obesity and overweight. World Health Organization. https://www.who.int/news-room/fact-sheets/ detail/obesity-and-overweight. Accessed 3 Feb 2021

27. Kaufman J, Stoddard J (2020) Coronavirus Impact Scale. https:// disasterinfo.nlm.nih.gov/search/? source $=2587$

28. van der Vijver F, Leung K (1997) Methods and data analysis for cross-cultural research. Sage Publications, London

29. Antony MM, Cox BJ, Enns MW et al (1998) Psychometric properties of the 42-item and 21-item versions of the Depression Anxiety Stress Scales in clinical groups and a community sample. Psychol Assess 10:176-181. https://doi.org/10.1037/1040-3590.10.2.176

30. Pais-Ribeiro JL, Honrado A, Leal I (2004) Contribuição para o estudo da adaptação portuguesa das escalas de ansiedade , depressão e stress (EADS) de 21 itens de Lovibond e Lovibond. Psicol saúde doenças 5:229-239. http://hdl.handle.net/10216/6910

31. Cappelleri JC, Bushmakin AG, Gerber RA et al (2009) Psychometric analysis of the Three-Factor Eating Questionnaire-R21: results from a large diverse sample of obese and non-obese participants. Int J Obes 33:611-620. https://doi.org/10.1038/ijo.2009. 74

32. Duarte PAS, Palmeira L, Pinto-Gouveia J (2020) The Three-Factor Eating Questionnaire-R21: a confirmatory factor analysis in a Portuguese sample. Eat Weight Disord Stud Anorexia Bulim Obes 25:247-256. https://doi.org/10.1007/s40519-018-0561-7

33. Anderson JC, Gerbing DW (1988) Structural equation modeling in practice: a review and recommended two-step approach. Psychol Bull 103:411-423. https://doi.org/10.1037/0033-2909.103.3.411

34. Hu L, Bentler PM (1999) Cutoff criteria for fit indexes in covariance structure analysis: conventional criteria versus new alternatives. Struct Equ Model A Multidiscip J 6:1-55. https://doi.org/ 10.1080/10705519909540118

35. Schumaker RE, Lomax RG (2010) A beginner's guide to structural equation modeling, 2nd edn. Routledge, New York

36. Deroost N, Cserjési R (2018) Attentional avoidance of emotional information in emotional eating. Psychiatry Res 269:172-177. https://doi.org/10.1016/j.psychres.2018.08.053

37. Hepworth R, Mogg K, Brignell C, Bradley BP (2010) Negative mood increases selective attention to food cues and subjective appetite. Appetite 54:134-142. https://doi.org/10.1016/j.appet. 2009.09.019

38. Wang C, Pan R, Wan X et al (2020) Immediate psychological responses and associated factors during the initial stage of the 2019 coronavirus disease (COVID-19) Epidemic Among The General Population in China. Int J Environ Res Public Health 17:1729. https://doi.org/10.3390/ijerph17051729

39. Özdin S, Bayrak Özdin Ş (2020) Levels and predictors of anxiety, depression and health anxiety during COVID-19 pandemic in Turkish society: the importance of gender. Int J Soc Psychiatry 66:504-511. https://doi.org/10.1177/0020764020927051

40. Daly M, Robinson E (2020) Psychological distress and adaptation to the COVID-19 crisis in the United States. J Psychiatr Res. https://doi.org/10.1016/j.jpsychires.2020.10.035

41. Vindegaard N, Benros ME (2020) COVID-19 pandemic and mental health consequences: systematic review of the current evidence. Brain Behav Immun 89:531-542

42. Gregório MJ, Irving S, Teixeira D et al (2021) The national food and nutrition strategy for the Portuguese COVID-19 response. Eur J Clin Nutr. https://doi.org/10.1038/s41430-020-00818-w

43. Direção Geral da Saúde (2020) REACT-COVID—Inquérito sobre Alimentação e Atividade Física em contexto de contenção social. Direção Geral da Saúde. https://www.dgs.pt/documentos-e-publi 
cacoes/inquerito-sobre-alimentacao-e-atividade-fisica-em-conte xto-de-contencao-social-pdf. Accessed 3 Feb 2021

44. Bhutani S, Cooper JA (2020) COVID-19 related home confinement in adults: weight gain risks and opportunities. Obesity. https://doi.org/10.1002/oby.22904

45. Hajifathalian K, Kumar S, Newberry C et al (2020) Obesity is associated with worse outcomes in COVID-19: analysis of early data from New York City. Obesity. https://doi.org/10.1002/oby. 22923

46. Zhou X, Snoswell CL, Harding LE et al (2020) The role of telehealth in reducing the mental health burden from COVID-19. Telemed e-Health 26:377-379. https://doi.org/10.1089/tmj.2020. 0068

47. Minihan E, Gavin B, Psychiatry A et al (2020) Covid-19, mental health and psychological first aid. Ir J Psychol Med. https://doi. org/10.1017/ipm.2020.41
48. Guo Q, Zheng Y, Shi J et al (2020) Immediate psychological distress in quarantined patients with COVID-19 and its association with peripheral inflammation: a mixed-method study. Brain Behav Immun. https://doi.org/10.1016/j.bbi.2020.05.038

49. Hayes A (2018) Introduction to mediation, moderation, and conditional process analysis, 2nd edn. The Guildford Press, New York

50. Smyth JM, Wonderlich SA, Crosby RD et al (2001) The use of ecological momentary assessment approaches in eating disorder research. Int J Eat Disord 30:83-95. https://doi.org/10.1002/eat. 1057

Publisher's Note Springer Nature remains neutral with regard to jurisdictional claims in published maps and institutional affiliations. 